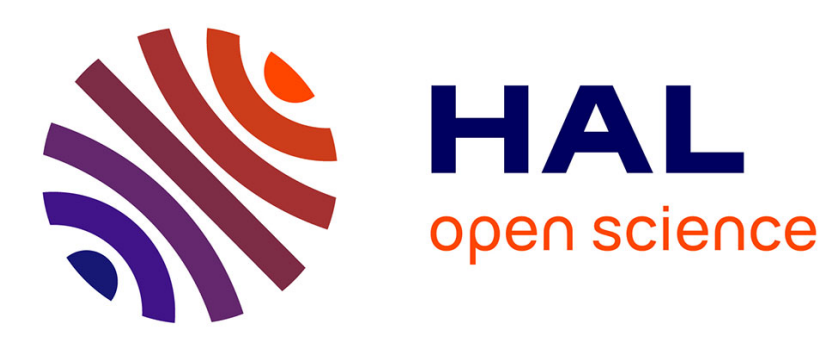

\title{
Merveille et conflits de valeurs dans les premiers textes de langue d'oïl : quelques exemples
}

\author{
Éléonore Andrieu
}

\section{To cite this version:}

Éléonore Andrieu. Merveille et conflits de valeurs dans les premiers textes de langue d'oïl : quelques exemples. F. E. Consolino, F. Marzella, L. Spetia éds. Aspetti del meraviglioso nelle letterature medievali. Medioevo latino, romanzo, germanico e celtico, Brepols, pp.137-148, 2016. hal-02148266

\section{HAL Id: hal-02148266 \\ https://hal.science/hal-02148266}

Submitted on 5 Jun 2019

HAL is a multi-disciplinary open access archive for the deposit and dissemination of scientific research documents, whether they are published or not. The documents may come from teaching and research institutions in France or abroad, or from public or private research centers.
L'archive ouverte pluridisciplinaire HAL, est destinée au dépôt et à la diffusion de documents scientifiques de niveau recherche, publiés ou non, émanant des établissements d'enseignement et de recherche français ou étrangers, des laboratoires publics ou privés. 


\section{MERVEILLE ET CONFLITS DE VALEURS DANS LES PREMIERS}

\section{TEXTES DE LANGUE D’Ö̈L : QUELQUES EXEMPLES}

Nous proposons d'envisager la notion de merveille comme un mécanisme susceptible de révéler la confrontation de différents systèmes de valeur dans les premiers textes littéraires de langue romane. La merveille est en effet source de polyphonie dans un énoncé, en raison de ce qui constitue sa «composante logico-sémantique », à savoir sa capacité à incarner «l'idée d'altérité », et à mettre en scène dans la narration « la perception d'un écart entre une expérience ou une opinion commune et l'événement inattendu qui se produit ${ }^{1} \gg$. Pour ce faire, le texte peut utiliser l'immédiateté du commentaire du narrateur, qui désigne au lecteur ce qui doit échapper à son système d'interprétation ordinaire. Il peut aussi mettre en scène un personnage, plus précisément la capacité du personnage à accepter l'étonnement, à percevoir la merveille et à refuser le secours de son propre système d'interprétation. Quand donc la narration, épique ou romanesque, accueille les composantes du merveilleux et plus encore, quand elle rend compte de la perte du sens immédiat de ces composantes en signalant l'apparition de la merveille, c'est bien alors, par-delà la rupture d'un horizon d'attente et des valeurs et normes qu'il contient, la mise en scène d'un autre discours interprétatif sur le monde qui devient perceptible. Mais il y a plus : la mise en scène d'une brisure dans l'interprétation assurée des choses du monde permet d'emblée de hiérarchiser en valeur les différents systèmes d'interprétation en présence et les acteurs placés devant la merveille. Et ce, en fonction de leur mode de réception. En effet, et comme l'ont montré les travaux de Francis Dubost ${ }^{2}$ et Jean-René Valette ${ }^{3}$, la merveille est un point de départ dynamique qui appelle une résolution sur deux plans : elle donne à l'extra-ordinaire une qualification éthique indiscutable (elle est l'expression du «plus haut degré » sur le plan évaluatif et axiologique) et au spectateur de l'extra-ordinaire, une compétence plus ou moins grande à y accéder. Ainsi, la capacité à percevoir la merveille conditionne la capacité du chevalier à entrer dans un itinéraire véritablement qualifiant, tandis que le lecteur, invité par le commentaire compétent du narrateur à la contempler, est d'emblée guidé vers ce qui revêt la plus haute valeur.

Une telle mise en scène de la subjectivité confrontée aux manifestations de l'extraordinaire n'a rien d'inédit. On la retrouve par exemple dans l'hagiographie ou dans l'historiographie ecclésiastiques depuis le très haut Moyen Âge, comme le montre par exemple Jacques Fontaine à propos des glissements de sens du mot miraculum dans la Vie de Saint Martin de Sulpice-Sévère ${ }^{4}$, depuis la réception-identification immédiate jusqu'à la suspension de l'identification, voire l'étonnement paralysant. Peter Brown a constaté que Grégoire de Tours apporte le même soin à la configuration des compétences face à l'extraordinaire : l'évêque de Tours hiérarchise deux sortes d'interprétation, deux sortes de systèmes d'explication, et deux sortes d'hommes. Il met ainsi en scène une réception marquée par la rusticitas, soit «l'échec, ou le refus positif, de structurer la vie en termes de relations

\footnotetext{
${ }^{1}$ J.-R. VAlette, dans Le Merlin de Robert de Boron, S. BAZIN-TACChElla, T. ReVol, J.-R. VAlETte, Neuilly-sur-Seine, Atlande, 2000, p. 69.

${ }^{2}$ Aspects fantastiques de la littérature narrative médiévale (XII ${ }^{e}$-XIII siècles). L'Autre, l'Ailleurs, l'Autrefois, Paris, Champion, 1991, 2 vols.

${ }^{3}$ La Poétique du merveilleux dans le Lancelot en prose, Paris, Champion, 1998, surtout les chapitres 1 à 3.

${ }^{4}$ Sulpice Sévère. Vie de saint Martin, t. I. Introduction, texte et traduction par J. FonTAINE, Paris, éd. du Cerf, 1967 ; t. II-III. Commentaire et Index par J. FonTAINE, Paris, éd. du Cerf, 1968-1969 (Coll. « Sources chrétiennes », $\mathrm{n}^{\circ}$ 133-135). Sur le miraculum, voir plus précisément t. II, p. 727-728 (pour les paysans pétrifiés) et surtout p. $756 s q$., qui explicite les traductions possibles du mot.
} 
avec des points de repère spécifiques et surnaturels $s^{5} »$, en raison de systèmes d'explication alternatifs que le récepteur de l'extraordinaire refuse ou est incapable d'écarter. Plus tard, au $\mathrm{XI}^{\mathrm{e}}$ et au XII ${ }^{\mathrm{e}}$ siècles, Bernard d'Angers ou Suger dénoncent dans les mêmes termes ceux qui ne parviennent pas à requalifier l'extraordinaire en termes spirituels. Comme l'a montré l'analyse précise de Dominique Barthélemy ${ }^{6}$, Bernard d'Angers, en relevant le terme jocum utilisé par les habitants de Conques à propos d'un miracle de sainte Foy, soupçonne une interprétation de l'extraordinaire qu'il récuse et dont il rend responsable le rusticus intellectus des témoins, en proposant de remplacer le terme par l'expression « miracle de minimis ». De fait, Bernard souligne que le danger le plus grand est le défaut de perception et d'interprétation des signes étonnants envoyés par Dieu, surtout quand ce signe ressemble à un « jeu ». Face à ce premier mode de réception, qui disqualifie la compétence des témoins, il est en effet une réception modelée par la reverentia ${ }^{7}$ qu'exaltent Sulpice-Sévère, Grégoire de Tours, Bernard d'Angers ou Suger, mais aussi bien le Livre de Job et ses mirabilia. Nous citerons à ce propos la synthèse remarquable de $\mathrm{P}$. Brown :

\begin{abstract}
La rusticitas, c'était donc le refus de voir le monde comme intelligible; et la reverentia, c'était la reconnaissance tacite que la vie est intelligible dans des termes posés [par Grégoire] sans la moindre équivoque. En bref : un monde rendu intelligible en termes de reverentia était un monde où la chance et la malchance étaient pensées comme autant de conséquences directes et tangibles de la rémission ou de la rétribution du péché. L'œuvre de Grégoire, en somme, émerge comme le point culminant de la tentative faite par des générations de prédicateurs chrétiens pour que la vie humaine, telle qu'on la vivait en Gaule, devienne explicable uniquement en termes de péchés et de conséquences du péché. C'était une tentative qui transcendait tous les schémas antérieurs d'explication ${ }^{8}$.
\end{abstract}

Il faut quoi qu'il en soit à la description de l'extraordinaire et de sa réception un dispositif énonciatif voyant, articulé sur une régie ferme (et visible) de l'univers textuel : l'identité du « je » qui met en scène la merveille, les regards sur la merveille et de la sorte, évalue les compétences, est en effet centrale dans ce dispositif ${ }^{9}$. Il est le point de vue autoritaire capable de percevoir et d'interpréter le spirituel dans l'extraordinaire et sa persona est très présente dans le récit de merveille. La figure de l'hypotypose se révèle particulièrement pertinente pour penser ce dispositif, surtout si l'on rappelle que la mise en récit de cette «description d'actions » peut avoir pour critères que «les marques, souvent énonciatives, donnent à comprendre que le tableau est une (re)création subjective: informations parcellaires, et faussement actualisées. C'est là toute l'ambivalence de l'hypotypose : on abolit la dimension figurative de l'énoncé tout en montrant qu'elle existe ${ }^{10} »$.

Or il se trouve que la spécificité de ce dispositif énonciatif s'ente sur un problème idéologique repérable dès les premiers énoncés mais dont les contours se précisent d'une façon particulière aux $\mathrm{XI}^{\mathrm{e}}$ et $\mathrm{XII}^{\mathrm{e}}$ siècles, en période grégorienne : il concerne l'assimilation, juridiquement argumentée dans la construction en cours de l’Église « rénovée », du «je » qui régit la reverentia au clerc. Ce qu'Yves Sassier ${ }^{11}$ appelle «l'auxiliarisation du laïc », surtout

\footnotetext{
${ }^{5}$ P. Brown, «Reliques et statut social au temps de Grégoire de Tours », repris dans La Société et le sacré dans l'Antiquité tardive, Paris, Le Seuil, 1985 (trad. frçse), p. 185-214, p. 191.

${ }^{6}$ «Un jeu de Sainte Foy de Conques : le miracle du faucon retrouvé (Bernard d'Angers, I.23) », dans Retour aux sources. Textes, études et documents d'histoire médiévale offerts à Michel Parisse, Paris, Picard, 2004, p. 383-394.

${ }^{7}$ P. BROWN, « Reliques et statut social au temps de Grégoire de Tours », p. 193.

${ }^{8}$ Ibid., p. 192-193.

${ }^{9}$ Voir, pour le texte littéraire, les précisions de J.-R. VALETTE, La Poétique du merveilleux, p. 217 sq.

${ }^{10}$ C. Fromilhague, Les Figures de style, Paris, Armand Colin, 1995, p. 107.

11 «Le début du XII ${ }^{\mathrm{e}}$ siècle est [...] le temps où fut exprimée de la façon la plus rigide l'idée d'une 'auxiliarité' de la fonction princière, notamment au travers d'une volonté tenace des partisans de la réforme
} 
du grand laïc, princes ou seigneurs, en découle: sur lui pèse en effet la menace de la rusticitas, sauf à recourir à la médiation du clerc comme dans la plupart des domaines de la vie sociale puisque telle est une des spécificités de la dite « Réforme ${ }^{12}{ }$... Autrement dit, la mise en scène du rapport, compétent ou non, aux signes merveilleux du spirituel ne peut que se confronter à la distinction des statuts et des rôles dans la société chrétienne que l'Église grégorienne s'attache à repenser: les itinéraires que suivent les différents regards et les différents modes de réception de la merveille sont en ce sens « socialement fondateurs ${ }^{13}$ ", dans la mesure où certains énoncés ecclésiastiques articulent le mirabilis aux compétences du regard de l'homme d'Église.

Il apparait, au travers de cette conjonction d'événements symboliques, politiques et idéologiques, que lorsque les premiers textes de langue d'oïl accueillent jusqu'aux termes mêmes de la merveille, ils n'utilisent pas un matériau neutre, ou universel, ou anachronique : du point de vue qui est le nôtre, ils accaparent un dispositif narratif extrêmement puissant et signifiant dans les énoncés ecclésiastiques contemporains. Ce mécanisme permet de postuler un principe dynamique de continuum entre les domaines d'écriture, non une simple opposition, pas davantage qu'une transparente reprise intertextuelle. Autrement dit, les textes dits « littéraires » participeraient pleinement aux débats sur la hiérarchisation des regards et des genres d'hommes placés face aux manifestations du spirituel sur la terre. Plus précisément, la confrontation « littéraire » d'un personnage, du lecteur et de la merveille serait un des lieux qui permettrait au texte littéraire de jouer pleinement sa partition dans les conditions de possibilité d'un héroïsme spécifiquement «laïc », largement mis en question à la période grégorienne.

Nous ferons donc l'hypothèse que la mise en scène de la merveille permet, dans chaque univers littéraire, de requalifier une des composantes de cet héroïsme contesté en lui donnant un caractère spirituel indiscutable. Dans le même mouvement, le texte littéraire confronterait le caractère exceptionnel de cet héroïsme à ce que disent du grand laïc les énoncés réformateurs : la merveille serait le moyen de hiérarchiser, cette fois au profit des grands laïcs, de multiples doxa. Dans les énoncés ecclésiastiques de la période, l'utilisation de la merveille permet la confrontation et la hiérarchisation des systèmes de valeurs au profit de l'accaparement du spirituel et de ses signes par l'homo spiritualis et de la disqualification de tous les autres systèmes d'explication alternatifs, dont celui du laïc, condamné à recourir à la médiation du clerc pour tout accès (perception/réception/interprétation) au spirituel. Mais quand la merveille glisse dans les nouveaux textes, quels sont les systèmes de valeurs disqualifiés, ou au contraire exaltés ? Il y aurait, de fait, une composante polémique, dialogique dans la mobilisation de la merveille par les nouveaux textes de langue d'oïl. Nous prendrons l'exemple de trois corpus pour appréhender, à défaut de les résoudre, ces questions complexes.

\section{I- LA MERVEILLE DES FAITS D’ARMES}

Dès les premiers textes de langue d'oïl, soit la production épique, le vocabulaire de la merveille fait son apparition avec insistance. Daniel Poirion ${ }^{14}$ précise que merveillos

d'affirmer le rôle moteur de l'Église dans la prise en charge de la paix publique, et de confiner rois et princes dans une simple fonction d'exécution » («L'Histoire du monastère de Vézelay de Hugues de Poitiers, reflet des tensions idéologiques du XII ${ }^{\mathrm{e}}$ siècle », Structures du pouvoir, royauté et Res Publica (France, IX ${ }^{e}-X I I^{e}$ siècle), Rouen, PURH, 2004, p. 163-175, p. 165).

${ }^{12} \mathrm{Cf}$. par exemple sur ce problème de la médiation : D. IOGNA-PRAT, Ordonner et exclure. Cluny et la société chrétienne face à l'hérésie, au judaïsme et à l'islam (1000-1150), Paris, Aubier, 1998.

${ }^{13}$ M. Goullet, D. IognA-PRAT, «La Vierge en Majesté de Clermont-Ferrand », Marie. Le culte de la Vierge dans la société médiévale, D. Iogna-Prat, E. PALAZzO, D. Russo (éd.), Paris, 1996, p. 383-405, p. 404.

14 « Théorie et pratique du style au Moyen Âge : le sublime et la merveille », RHLF, I (1986), p. 15-22, p. 24. 
concurrence grant et halt pour «marquer cette qualité étonnante de la grandeur épique »... Plus largement, la merveille épique s'applique avec constance au domaine de la fonction guerrière : armes, faits d'armes, acteurs des faits d'armes, vertus propres du héros guerrier ${ }^{15}$.

Or, précisément, la fonction guerrière a servi, dans certains énoncés ecclésiastiques clunisiens puis réformateurs, d'argument fondamental à la disqualification éthique du grand laïc : réduit à sa mission guerrière, le «prince de la terre "s'est vu confier, dès le texte de l'Astronome et celui de Jonas d'Orléans, la régulation des conflits dans la cité terrestre, au détriment de toutes les autres missions du "prince chef de l'Église » qu'accaparaient jusqu'alors les personnages de grands laïcs dans les biographies carolingiennes, par exemple celles d'Eginhard ou d'Ermold le Noir ${ }^{16}$. L'auxiliarisation du groupe des « grands laïcs » qui en découle est sans précédent à l'époque grégorienne. Le sacre tel qu'Innocent III est parvenu, en 1204, à le mettre en place, synthétise cette évolution : désormais, c'est la main et le bras du prince qui seront oints, non le cœur, comme pour les prélats. Les Vitae des princes mettent en lumière ce qu'Alain Boureau appelle une «conception purement et agressivement profane », « une conception contractuelle ou utilitariste ${ }^{17}$ », non seulement de la royauté, mais aussi plus généralement de l'ensemble du groupe des grands laïcs. Or, certains énoncés ecclésiastiques ne répugnent pas, loin de là, à mobiliser une écriture de la merveille épique, appliquée donc à la fonction guerrière des grands laïcs ${ }^{18}$. On prendra l'exemple des Gesta Ludovici Grossi de Suger, consacrés exclusivement au récit des guerres du roi Louis VI contre ses vassaux et voisins récalcitrants ${ }^{19}$, et où l'on peut noter la présence insistante de l'extraordinaire. Mais la mise en scène du regard sidéré y témoigne en réalité de la valeur éthique relative de la sphère épique dans laquelle évoluent à la fois le héros, ses adjuvants et ses opposants aussi bien ${ }^{20}$. Suger précise ainsi en distinguant sa propre compétence de la leur que pour ses personnages de « grands laïcs », la guerre est un événement douteux (ut dubius se habet belli eventus ${ }^{21}$ ) et qu'une roue tourne qui entraîne avec elle succès et défaite ${ }^{22}:$ ils ignorent le dessein divin qui

${ }^{15}$ Cf., outre l'analyse de J.-R. VAlette dans La Poétique du merveilleux, N. AndRIEUX-ReIX, Ancien Français. Fiches de vocabulaire, Paris, PUF, 1987, notamment la fiche consacrée au mot « preux ».

${ }^{16} \mathrm{D}$. Iogna-Prat a démontré la transformation de la conception que l'on peut dire « cléricalisée » du roi filius ecclesiae, dès lors opposée à la théorie d'un roi rector ecclesiae, dans « La construction biographique du souverain carolingien », À la recherche de légitimités chrétiennes. Représentations de l'espace et du temps dans l'Espagne médiévale (IX - XIII siècle), P. HENRIET (dir.), Annexes des cahiers de linguistique et de civilisation hispaniques médiévales, vol. 15 (2003), Lyon, p. 197- 224 (repris et remanié dans La Maison Dieu. Une histoire monumentale de l'Église au Moyen Âge (v. 800-v. 1200), Paris, éd. du Seuil, 2006, p. 119 sq.). Ainsi, Alcuin, Paulin d'Aquilée, Théodulphe d'Orléans, Smaragde de Saint-Mihiel, Ermold le Noir, Eginhard, Charlemagne et Louis le Pieux exaltent encore la potestas d'un roi rex et sacerdos, responsable devant Dieu seul. Le roi unit en lui, dans ces versions «monarchiques » de la potestas, l'orator et le bellator. A contrario, et à la suite de Jonas d'Orléans, la biographie de Louis le Pieux par l'Astronome transforme vers 840 le personnage du bon roi et impose à ses côtés, symptomatiquement, des personnages de clercs, dont le narrateur lui-même qui, comme son surnom le montre, devient le seul lecteur autorisé des signes du ciel devenus inaccessibles à la « ministérialité » royale. Dès lors, la fonction guerrière (illustrée par Charlemagne chez l'Astronome) subit une dégradation sans précédent.

17 «Un obstacle à la sacralité royale en Occident : le principe hiérarchique », La royauté sacrée dans le monde chrétien, A. BoureAU et C. S. INGERflom (dir.), Paris, éd. de l'EHESS, 1992, p. 29-37, p. 30.

${ }^{18}$ Voir D. BARTHELEMY, Chevaliers et miracles. La violence et le sacré dans la société féodale, Paris, Armand Colin, 2004.

${ }^{19}$ La Vie de Louis VI le Gros, éd. et trad. d'H. WAQUET, Paris, Les Belles Lettres, 1929 (coll. «Les Classiques de l'Histoire de France au Moyen Âge »).

${ }^{20}$ Nous nous permettons de renvoyer pour une analyse plus complète du lexique et une bibliographie à notre article : «Le regard stupéfait, la beauté et la mise en ordres dans les textes de Suger », La beauté du merveilleux, A. GAILlARD et J.-R. VALETTE éd., Pessac, PUB, 2011, p. 111-141. Nous reprenons ici une partie des conclusions.

${ }^{21}$ Vie de Louis VI, I, p. 8

${ }^{22}$ C'est la même chose dans le récit qui concerne le Puiset, avec une reprise de la formule : Se habet belli dubius eventus (21, p. 168). A propos du comte du Puiset de summo rote exorbitans declinando, tandis que 
les mène. C'est, précisément, cette ignorance qui produit la merveille et lui donne un sens : les personnages et le lecteur dans un premier temps sont subjugués par la force guerrière et le récit des beaux combats parce qu'ils ne peuvent guère voir plus loin et de fait, déceler ce qui est vraiment extraordinaire. Autrement dit, Suger présente des personnages incapables de voir à l'œuvre un principe purement spirituel, exactement comme le fera Joseph d'Exeter dans l'Ylias en narrant la guerre de Troie ${ }^{23}$. Et il prend soin de ménager une régie narrative bien supérieure en compétence (la sienne) par rapport aux regards sidérés par l'extraordinaire épique : une analyse exhaustive du vocabulaire du merveilleux non seulement dans la Vie de Louis VI mais aussi bien dans ses autres œuvres ${ }^{24}$ montre la rupture entre cette régie narrative et les acteurs laïques de l'Histoire. Suger construit le regard de l'historien (historiographus) ecclésiastique qui a la compétence nécessaire pour lire, derrière le chaos guerrier, la main de Dieu et donc, le véritable extraordinaire (relative au miraculus). Dès lors, qui perçoit la merveille épique dévoile au fond un regard aveuglé, infirme, incompétent, sidéré par une forme de surhumanité provisoire, factice... Le narrateur joue donc avec le caractère épique et sa matière : il manipule un regard stupéfait par l'extraordinaire de nature épique pour mieux le disqualifier et relancer la lecture de l'intervention divine et surtout, du rôle médiateur essentiel des clercs. Dans le De consecratione, Suger explicite fort clairement, en citant Lucain pour mieux l'écarter, cette loi de composition de son texte épique et d'utilisation de l'étonnement « merveilleux »:

Secutum est aliud dignum memoria factum, quod non ex accidenti, sicut de talibus judicant qui illi consentiunt secte, videlicet quod : "Fors incerta vagatur,/ Fertque refertque vices, et habent mortalia casus ${ }^{25}$ sed divina largitione (Un autre événement digne de mémoire survint alors, non par l'effet du hasard, comme jugent de ces choses ceux qui adhèrent à cette doctrine selon laquelle « Le sort erre sans but,/ Il emporte et rapporte toutes choses,/ Et les hasards disposent de tout ce qui est mortel ». Mais [ce fut] par l'effet de la largesse divine) (XII, p. 38).

Ce que les hommes qui combattent prennent pour la roue du destin ou l'expression du hasard, en s'en étonnant, n'est que le résultat de leur aveuglement et/ou de leur éloignement par rapport au spirituel, seule source du véritable émerveillement, pris en charge par l'abbé. La fonction guerrière choisie à l'exclusion de tout autre pour représenter le roi Louis VI donne certes lieu à une théâtralisation de nature épique, qui étonne le lecteur stupéfait d'y retrouver cette perfection héroïque de l'épopée et des chansons de geste contemporaines. Mais elle ne véhicule que l'incomplétude d'un prince laïc sans véritable grandeur comme en témoigne la lecture parallèle des autres écrits de l'abbé : comme l'avait souligné M. Heinzelmann à propos de l'œuvre de Grégoire de Tours ${ }^{26}$, la lecture du seul pan «historiographique » du corpus de tels narrateurs n'est pas en accord avec la cohérence extrême (ce qui n'exclut pas du tout leurs ambiguïtés) de leur système de représentation. Par la confrontation avec le pan

le roi affirme sa force (21, p. 168). A propos du roi d'Angleterre: Rex Anglie, post longos et mirabiles placidissime prosperatis successus, quasi de summo rote descendens, mutabili et infausto rerum angariatur eventu...(26, p. 188).

${ }^{23}$ Selon les analyses de F. Mora. Voir sur ce texte (L'Iliade. Epopée du XII e siècle sur la guerre de Troie, traduction et notes sous la direction de F. Mora, introduction de J.-Y. Tilliette, Turnhout, 2003), les nombreuses analyses de F. Mora : «Galerie de portraits et tombeau de Teuthras dans le livre IV du De Bello Trojano de Joseph d'Exeter : la perfection insaisissable », revue PRIS-MA, XVI/2 (2000), p. 249-265; «L'Ylias de Joseph d'Exeter : une réaction cléricale au Roman de Troie de Benoît de Sainte-Maure », Progrès, réaction, décadence dans l'occident médiéval, E. Baumgartner et L. Harf-Lancner éd., Genève, p. 199-213.

${ }^{24}$ Euvres de Suger, texte établi, traduit et commenté par F. GASPARRI, Paris, Champion, t. I, 1996 et t. II, 2001 (Coll. «Les Classiques de l'histoire de France au Moyen Âge »).

${ }^{25}$ Lucain, La Pharsale, II, 13.

${ }^{26}$ Gregory of Tours. History and Society in the Sixth Century, Cambridge/ New-York, Cambridge University Press, 2001 (trad.). 
«administratif» de son œuvre, le merveilleux épique dont Suger nourrit abondamment ses écrits sur le roi s'en trouve dévalué au profit d'un mirabilis produit et contrôlé par les clercs et seul véhicule vers le supérieur et la beauté qui en est le signe : c'est ce que montre le $D e$ consecratione, tout particulièrement, consacré à la basilique et à l'action des clerici de l'abbaye.

Il est intéressant dès lors de confronter cette merveille épique dévaluée, disqualifiée, à la merveille épique des chansons de geste, qui qualifie la même matière guerrière. Le texte dit littéraire remodèlerait ainsi, au moyen des merveilloses batailles, un personnage de grand laïc que l'énoncé ecclésiastique disqualifiait à travers, précisément, sa fonction guerrière : le mouvement de reprise du même motif (la fonction guerrière) s'accompagne d'un mouvement d'écart polémique, puisqu'au lieu de faire de la merveille guerrière le signe d'un regard aveuglé et d'une surhumanité factice, dénoncés par un regard ecclésiastique, la chanson de geste en fait une valeur éthique garantie et reconnecte le fait d'armes ainsi qualifié au spirituel. Le chevalier de la chanson de geste, martyr comme Roland ou Vivien, ou bien combattant comme Rainouart ou Guillaume, bénéficie d'un accès direct au spirituel du sein même de son acte guerrier, sans conversion à l'état clérical et sans causalité cléricale originelle (il n'est point d'appel à la guerre sainte dans les chansons). Ce que révèlerait la merveille épique, c'est bien le sens profondément spirituel de la guerre et du combat, sens que Dieu confère directement aux événements sans plus en passer par la main et la parole du prélat. Ainsi, la merveille est le signe pour celui qui en est l'acteur -ici le chevalier combattant - d'une plus haute valeur, de nature indéniablement spirituelle.

Ce que la mise en scène de la merveille épique révèle aussi, c'est la confrontation polémique et la hiérarchisation des discours et des doxa au sein de ce processus: les monstrations appuyées et insistantes de l'extraordinaire au sein de la diégèse épique consistent littéralement à mettre à l'écart, à désactiver une «opinion commune », selon la définition même de la merveille. Or cette opinion commune disqualifiée, désactivée et impuissante à rendre compte de la plus haute valeur du guerrier, c'est bien le discours grégorien sur la fonction guerrière, sur l'évaluation de la guerre et de la mission du laïc. La merveille désigne avec insistance ce qui rompt avec cette doxa, soit une qualification véritablement spirituelle de la fonction guerrière. Autrement dit, mettre en scène la merveille épique consiste à tenir un véritable discours de contestation de l'auxiliarisation de la fonction guerrière dans le discours ecclésiastique. Nous pouvons sur ce point citer D. Poirion : « La démesure, le dépassement héroïque conduisant à un excès de morts, la rencontre d'un surnaturel qui n'est pas dans l'exact prolongement du geste sublime, préparent la mise en question par la littérature profane, en langue vulgaire, sous le couvert du merveilleux, de la grandeur $^{27} »$. Et le terme même qui est choisi préférentiellement par le texte littéraire, merveille, est bien peut-être la preuve, par rapport au choix toujours possible en ancien français du terme rare de miracle, que la spiritualisation de l'acte guerrier configurée ici est désinstitutionnalisée, décléricalisée. Au point que même le «miracle» de la vision de Guillaume, dans le Charroi de Nîmes, ne bénéficie pas d'une telle qualification...

\section{II- LA MERVEILle D'YVAIN ET CAlogrenant : UNE QUESTION DE}

\section{GOUVERNEMENT}

Chrétien de Troyes fournit, dans le Chevalier au lion, une autre mise en scène de la merveille très intéressante pour notre propos: il dispose de part et d'autre de la scène étonnante deux regards différents, donc deux systèmes d'explication qu'il hiérarchise puisque l'un est condamné, et l'autre qualifié de la plus haute valeur éthique.

\footnotetext{
${ }^{27}$ D. POIRION, « Théorie et pratique du style au Moyen Âge », p. 24.
} 
Devant un gardien de taureaux gigantesque et extraordinaire, Calogrenant, le chevalier le «plus avenant et le plus courtois » de la cour d'Arthur, semble ne pas pouvoir renoncer à un système d'explication extrêmement précis, qui le conduit à interpréter les objets rencontrés selon les catégories qui sont les siennes: animalisation, marginalisation, vilenie ${ }^{28}$. Plus précisément, il se montre incapable de concevoir, même en le voyant, que le gardien puisse contenir sans lien et sans attache des bêtes sauvages dans un endroit précis et qu'il exerce sur elles un pouvoir de «seigneurie». De fait, Calogrenant n'emploie jamais le terme de merveille dans son discours direct : il ne semble pas apte à entrer dans un processus de déprise et d'incompréhension sans évaluation, susceptible de marquer une soumission au moins provisoire à ce qui dépasse sa propre doxa et lui résiste. Par opposition à ce premier personnage, Yvain est représenté comme étant soumis à un désir hors de raison et farouchement individuel d'aventure vers le «vilain» de Calogrenant ${ }^{29}$ : dès lors qu'il se trouve placé face à la scène extraordinaire, il se signe et qualifie cette fois sans hésitation la merveille $^{30}$. L'ébahissement d'Yvain, lecteur compétent de la merveille, suspend toute interprétation évaluative et axiologique. Yvain, est-il besoin de le préciser, entre dès lors, par cette première étape dans un itinéraire qualifiant qui le conduit, comme d'autres personnages chevaleresques de Chrétien de Troyes, à la conquête d'une valeur pleinement spirituelle là encore : la joie de l'amor fine. Le lecteur peut donc repérer la haute valeur de cet itinéraire par la capacité du personnage à désirer puis à accepter sans l'évaluer le caractère extraordinaire d'un événement ou d'un objet.

Calogrenant est un personnage d'autant plus complexe qu'il manifeste face à l'extraordinaire deux doxa entrelacées et toute deux également disqualifiées par la scène de l'essart : une doxa que les instructions données par les vers de l'incipit du roman nous incite à qualifier de courtoise, ce qui conduit le personnage à appliquer au paysage de l'essart, comme à son envers absolu, la qualification de vilenie. De la sorte, le gardien de taureaux apparaît avant tout comme un être sauvage, inapte à répondre soit par les armes au rituel chevaleresque de l'affrontement, soit par la parole au regard insistant de son interlocuteur. Mais la merveille permet de percevoir une seconde doxa dans le regard de Calogrenant : après avoir condamné la doxa courtoise, elle condamne aussi une doxa ecclésiastique, plus précisément réformatrice. Cette fois, ce n'est pas l'énoncé sur la fonction guerrière du laïc qui est en jeu, mais celui qui porte sur la forme de la domination seigneuriale laïque ${ }^{31}$. Ainsi, dans l'essart, il est bien question du mode de gouvernement des taureaux par leur «sire». Calogrenant est plus précisément invité à reconnaître comme extraordinaire, et comme doté d'une haute valeur de nature spirituelle, l'efficacité sociale et juridique de la force coercitive. Mais il refuse littéralement de voir dans l'essart que l'exercice violent de la force physique, source de crainte, est au fondement de la constitution d'un troupeau stable, obéissant à une loi extérieure à lui. Pour lui, la violence des bêtes ne réclame que la clôture et le lien et ne saurait aboutir à la formation du troupeau autour de son gardien : c'est une forme de pastoralisme inédit pour lui. Autrement dit, Calogrenant, chevalier de la fin du XII ${ }^{\mathrm{e}}$ siècle, a une identité culturelle qui ne lui permet pas de concevoir la valeur fondatrice et la nécessité de la force physique et de la violence, à la préhistoire de toute société humaine, à la source de toute justice, de toute paix et de toute joie... Or, cette identité culturelle est très précisément celle qui préside aux

${ }^{28}$ Le Chevalier au Lion (Yvain), Les Romans de Chrétien de Troyes édités d'après la copie de Guiot (BN fr. 794), IV, éd. M. RoQUES, Paris, Champion, 1960.

${ }^{29}$ L'instauration du champ sémantique du désir d'Yvain, juste après le discours de Calogrenant, est très intéressante de ce point de vue.

${ }^{30}$ Plus de .c. fois se seigna / de la merveille que il ot (v. 794-795).

${ }^{31}$ Nous nous permettons de renvoyer, pour une démonstration plus complète, à notre article : «Si le destraing par mi le cors : droit et violence dans l'essart (Le Chevalier au Lion de Chrétien de Troyes) », Droit et violence au Moyen Âge, P. HAUGEARD, M. OTT dir., Paris, Classiques Garnier, 2013, p. 39-51. C'est cette analyse que nous reprenons ici. 
interprétations et exégèses grégoriennes de la Genèse $e^{32}$, mais aussi à la constitution du droit canon grégorien et à la narration des vitae «réformatrices » de la période. Les clercs de la réforme grégorienne démontrent à longueur de pages, et jusque dans le texte de la Genèse, que la domination des princes est une mauvaise violence, bestiale, marquée du poids de la faute, de l'orgueil et perpétuant jusqu'au Jugement dernier une hiérarchie insoutenable. Le chevalier courtois Calogrenant, saturé de savoirs cléricaux, connaît bien le pouvoir pastoral, celui d'avant la Chute, dans lequel l'amour et l'humilité du berger suscitent l'obéissance absolue et l'amour des bêtes. Il connaît tout aussi bien la «domination pure », soit la dimension coercitive et orgueilleuse, incapable de conseil et de mansuétude, de la potestas concédée aux grands laïcs depuis la Chute. Mais de la sorte, il ne saurait concevoir que le gardien, non clerc, soit apte à rassembler un véritable troupeau, en une forme de pastoralisme inversé qui donne à la force du gardien la place et la valeur englobantes de l'amour du pasteur, au fondement du droit et de la société. La lecture de Calogrenant sur les composantes de la domination est clairement grégorienne et ne lui permet pas d'accepter l'altérité étonnante d'une autre doxa, seulement de la condamner d'emblée.

La merveille permet ici, comme la merveille épique, de mettre en lumière et de rendre incontestable en valeur une doxa différente de celle de certains énoncés ecclésiastiques de la période, qui nous semblent incarnés par Calogrenant dans la diégèse. En contexte, cette doxa valorisant la violence et la force, qu'avait repérée E. Baumgartner ${ }^{33}$, ne va pas de soi, mais se retrouve en d'autres énoncés : le roman propose de faire de la force coercitive, de la justitia, la source du lien social en activant les mêmes valeurs que celles des seigneurs soucieux de marquer par leur maîtrise du rituel de la chasse la valeur, mêlée de savoir et de violence, de leur pouvoir sur les bêtes et les hommes ${ }^{34}$. Après la scène de l'essart, la force et la violence seront placées dans le roman à la source même de l'amour fine, en une sorte de modèle inverse du gouvernement pastoral qu'accaparent les clercs et que connait trop bien Calogrenant. Chrétien place ainsi cette violence, à travers Yvain qui la voit et s'y soumet, en contact direct avec la boene pes, l'acorde, la fine amor. La merveille du gardien le prouve : il faut user de la crainte sur les sujets non seulement pour (r)établir le droit et adoucir les êtres malgré eux, comme le fait Arthur en son jugement de la querelle des deux sœurs ${ }^{35}$, mais aussi pour établir le lien social et l'amour, désiré cette fois... Yvain retrouve Laudine en frappant la fontaine par force et par estovoir (v. 6512) et en utilisant la férocité extrême du lion. Violence et droit, mais aussi violence et amour, violence et miséricorde, au fondement de la societas des hommes : la merveille inaugurale de l'essart donne le programme du roman tout entier et confère à l'héroïsme du personnage chevaleresque apte à la percevoir des contours propres à le distinguer à la fois du seigneur seulement courtois et du pasteur clérical.

Le roman fait ainsi, à travers la qualification d'une scène par le lexique de la merveille, l'éloge des signes de la violence, de la force, de la hiérarchie et de la justice punitive ${ }^{36}$. C'est ce que dit l'extraordinaire : la domination seigneuriale y trouve une justification sous la forme d'une valeur spirituelle garantie signalée par la merveille, y compris en ce qui concerne sa

\footnotetext{
${ }^{32}$ Voir P. BUC, L'Ambiguïté du Livre. Prince, pouvoir et peuple dans les commentaires de la Bible au Moyen Âge, Paris, Aubier, 1994.

${ }^{33}$ E. BAUMgartner, Romans de la Table Ronde de Chrétien de Troyes, Paris, Gallimard, 2003.

${ }^{34}$ Voir A. GUERREAU «Les structures de base de la chasse médiévale », A. PARAVICINI BAGLIANI et B. VAn Den AbeEle (éd.), La chasse au Moyen Âge. Sociétés, traités, symboles, Florence, Edizioni del Galluzzo, 2000, p. 25-32 ( coll. Micrologus 'Library) et P. BUC, L'Ambiguïté du Livre, p. 112-122.

${ }^{35}$ Il le dit por essaier/ S'il la porroit tant esmaier/ qu'ele randist a sa seror/ son heritage, par peor,/ qu'il s'est aparceüz molt bien/ que ele ne l'en randist rien/ por quan que dire li seüst/ se force ou crieme n'i eüst./ Por ce que ele dote et crient,/li dit : «Biax sire, or me covient/ que je face vostre talant » (v. 6415-6425).

${ }^{36}$ Même la violence du lion, conformément à ce qu'expliquent « les bestiaires médiévaux, [est] celle de l'animal assez sûr de sa domination pour savoir se maîtriser tout en utilisant quand il le faut une fureur et une force qui restent indispensables » (E. BAUMGARTNER, Romans de la Table Ronde, p. 116).
} 
composante coercitive. Le vrai héros chevaleresque, qui perçoit dans l'essart la première image du seigneur de la Fontaine qu'il est appelé à devenir hors de la cour seulement courtoise, échappe comme le signale sa perception de la merveille à une série de doxa attendues, et impuissantes à rendre compte de la spécificité merveilleuse : la doxa courtoise, la doxa grégorienne sur les formes de la domination. En entrant de plain-pied dans la reconnaissance de l'extraordinaire, le chevalier s'engage dans un itinéraire à la fois spirituel et décléricalisé qui le conduit à devenir celui qui, à la lettre, aime le mieux, tout en conservant des caractères charnels, seigneuriaux, et de fait, l'usage de la force et de la violence: autrement dit, son héroïsme rassemble scandaleusement, ou merveilleusement plutôt, des composantes soigneusement distinguées dans les énoncés grégoriens...

\section{III- LES « MIRACLES » DE RICHARD I ${ }^{\mathrm{er}}$}

L'Estoire des ducs de Normandie de Benoît de Sainte-Maure, si mal nommée Chronique des ducs de Normandie ${ }^{37}$, offre les mêmes possibilités d'interprétation de la merveille comme lieu de contestation et de mise à l'écart d'un énoncé ecclésiastique, et de spiritualisation positive de l'héroïsme laïc. C'est un corpus que sa proximité immédiate avec un autre texte, celui de Wace, dont on soupçonne que le travail fut interrompu vers 1170 par Henri II au profit du nouvel historien ${ }^{38}$, rend d'autant plus complexe.

Ce texte complexe, de nature historiographique, met en particulier en scène la merveille que constitue pour son narrateur trois épisodes de la vie du duc Richard Ier (v. 27185 sq.) : un combat contre le diable, une vision de pommier fertile au cœur de l'hiver, une histoire de sacristain concupiscent noyé, ressuscité et sauvé par l'intervention du duc. Françoise Laurent, qui a analysé très récemment ce triple récit dans le détail de son déroulement $^{39}$, en fait le signe de la captation par le personnage des «valeurs de la prestigieuse première fonction qui vient compléter et couronner les deux autres ${ }^{40} \gg$ fonctions indo-européennes. Ainsi, le personnage de grand laïc configuré par Benoît serait-il un prince idéal, dont la merveille constituerait comme l'indice incontestable de la valeur, en ce qu'elle marque le surgissement d'une fonction «spirituelle» du personnage : elle construirait son « exemplarité ». L'analyse de Françoise Laurent à propos de la merveille confirmerait donc son pouvoir narratif et idéologique particulier, cette fois dans le cadre historiographique.

Il semble possible d'ajouter à cette analyse une donnée supplémentaire, propre à préciser peut-être la spécificité de l'entreprise de Benoît: il se trouve que si le caractère merveilleux des épisodes que traverse Richard Ier suffit chez Benoît à le qualifier de « sainz », il n'est absolument pas question d'un tel qualificatif dans le texte de Wace. Wace évoque, dans son Roman de Rou, une «gaberie » au sujet de l'épisode du sacristain (v. 508) puis il fait de Richard Ier un «bon seignur» (v. 763). Il se pourrait, comme en témoigne cet épisode, mais bien plus largement d'autres indices de la structuration divergente de ces textes commentés en ce sens par Georges Duby ${ }^{41}$, que nous disposions avec le texte de Wace de la version ecclésiastique du texte de Benoît: le refus de Wace de qualifier les épisodes de

${ }^{37}$ Benoît de Sainte-Maure, La Chronique des Ducs de Normandie par Benoît, éd. C. FAHLIN, t. I et II, Uppsala, 1951-1954 (t. III : Glossaire revue et complété par O. SöDERGARD, Uppsala, 1967 ; t. IV : Notes, par S. SANDQVIST, Stockholm, 1979).

38 M. Aurell, L'Empire des Plantagenêt (1154-1224), Paris, Perrin, 2003, p. 152 sq. ; J.G. GoutTEBroze, «Pourquoi congédier un historiographe ? Henri II Plantagenêt et Wace (1155-1174)», Romania, 112 (1991), p. 239-311.

39 «Les anecdotes de la 'vie' de Richard Ier dans l'Histoire des ducs de Normandie de Benoît de SainteMaure », Furent les merveilles pruvees et les aventures truvees : hommage à Francis Dubost, F. GINGRAS, F. LAurent, F. Le NAN, J.-R. VAletTe dir., Paris, Champion, 2005, p. 369-392.

${ }^{40}$ Pour Dieu et pour le roi. Rhétorique et idéologie dans l'Histoire des ducs de Normandie de Benoît de Sainte-Maure, Paris, Champion, 2010, p. 228.

${ }^{41}$ Les trois ordres ou l'imaginaire du féodalisme, Paris, Gallimard, 1978, p. 331-337. 
merveilles pourrait bien servir à établir que si Wace a construit une vie des ducs de Normandie, il l'a fait à la manière de Suger, en leur refusant toute responsabilité, tout librearbitre et toute valeur éthique supérieure dans l'Histoire des hommes. On peut rappeler l'hypothèse de M. Aurell, selon lequel la «conception toute grégorienne » de la royauté de Wace aurait choqué son commanditaire, soucieux dès ce moment de le remplacer par Benoît, artisan d'une histoire des rois écrite au profit des rois et de leur action, et démontrant leur plus haute valeur, de nature spirituelle, tout en décléricalisant leur rapport au divin. Benoît de Sainte-Maure, comme les autres metteurs en scène de la merveille que nous avons rencontrés, opère bien la promotion éthique incontestable d'un personnage de grand laïc, en lui conférant un caractère spirituel. Mais si on le compare à ces autres metteurs en scène, si l'on rappelle le contexte d'écriture de la merveille et si l'on analyse l'écriture de Wace et son refus de la merveille, il semble possible d'ajouter que son écriture de l'histoire entre par là même en un rapport polémique, comme le montrent les merveilles de Richard Ier, avec l'écriture de l'histoire ecclésiastique dont la doxa est mise singulièrement à l'écart. En la Chronique des ducs de Normandie, Richard Ier opère lui-même la merveille, manifestant non pas tant la figure du prince idéal, ou la construction d'un « mythe », que la figure reconquise, en contexte Plantagenêt, d'un prince directement en lien avec le spirituel, sans aucune médiation cléricale : la rencontre entre Martin, abbé de Jumièges, et le duc Guillaume, telle que Benoît la traduit à partir de Dudon de Saint-Quentin est une autre déclinaison de ce glissement fondamental opéré par le récit et décelable dans l'utilisation de la merveille. G. Duby dans son commentaire démontrait qu'en ce passage, où c'est au prince laïc que revient de décrire l'ordre de la société, «le prince s'est [...] haussé jusqu'à ce degré supérieur, extérieur, qu'occupaient jadis les moines, Raoul Glaber ou saint Bernard, jugeant le siècle ${ }^{42} »$. Dès lors, effectivement, le prince domine et manifeste la merveille, autrement dit la fonction spirituelle. Et il ne semble pas qu'a contrario Wace se livre à une écriture «profane » ou «critique » de l'histoire, voire «légère », ou encore indépendante: bien au contraire. Il semble tenir fermement, face à Benoît de Sainte-Maure, à la démonstration de l'action de Dieu et des hommes de l'Église de Dieu dans l'Histoire, les rois et les grands n'étant que les instruments, à la surhumanité factice, de sa volonté et les exemples de son action rétributive. Pour discerner les discours, la merveille joue de fait admirablement son rôle de «désencodage ${ }^{43}$ » des discours et systèmes de valeur en présence, cette fois en contexte historiographique.

Le fait que nous puissions accorder ce sens à la merveille littéraire n'a rien de bien étonnant cependant : tous les textes évoqués sont a priori remarquables d'abord parce qu'ils sont le fruit d'un événement exceptionnel dans l'histoire des discours et de la société occidentale aussi bien. Cet événement a été déjà bien décrit par la sociolinguistique ${ }^{44}$, qui l'avait repéré au cœur de l'histoire de la langue : il s'agit de la captation, par l'aristocratie laïque, des signes attendus de la «littérarité » qu'accaparaient jusqu'alors les énoncés

${ }^{42}$ Les trois ordres, p. 333.

${ }^{43}$ Ce terme est emprunté à A. Guerreau-Jalabert, qui l'explicite par exemple dans « Fées et chevalerie. Observations sur le sens social d'un thème dit merveilleux », Miracles, prodiges et merveilles au Moyen Âge ; XXV Congrès de la S.H.M.E.S (Orléans, juin 1994), Paris, Publications de la Sorbonne, 1995 , p. $133-150$.

44 Outre les travaux de M. Delbouille (notamment « La formation des langues littéraires et les premiers textes », Grundriss der Romanischen Literaturen des Mittelalters, Heidelberg, 1972, t. 1, p. $560-584$ et p. 604-622), voir la mise au point de M. BANNIARD, «Genèse de la langue française (III ${ }^{\mathrm{e}}-\mathrm{X}^{\mathrm{e}}$ siècles) 》 dans F. Lestringant, M. ZINK (dir.), Histoire de la France littéraire, t. I : Naissances, Renaissances, Moyen Âge$X V I^{e}$ siècle, Paris, PUF, 2006, p. 9-35. 
ecclésiastiques, comme l'ont démontré M. Banniard ou encore A. Guerreau-Jalabert. Une telle « littérarité » est semble-t-il à entendre à la fois sur le plan éthique et sur le plan esthétique et elle a une lourde signification sociale : elle fait partie intégrante de l'identité d'un groupe social qui prétend à la conquête d'une certaine domination, ou qui prétend la défendre. Le groupe en quête de cette «littérarité », de ce «haut langage » pourvoyeur de valeurs, d'autorité, et d'une héroïcité réceptible pour ses membres et pour l'ensemble de la société, est soumis à une exigence double de «reprise » de formes, thèmes et valeurs dominants (on ne peut pas dire et imposer une valeur qui ne serait pas au moins en partie composée d'éléments collectivement perçus, dans la société chrétienne, comme nécessaires à cette valeur) et " d'écart », afin qu'il lui soit possible de marquer clairement la spécificité de son discours. La merveille serait donc un des rouages de cette construction de la valeur, rouage de monstration spectaculaire et polémique de la rupture avec une doxa ecclésiastique rendue proprement inefficace devant l'évidence d'un héroïsme laïc inédit : c'est littéralement quand cette doxa ne peut plus interpréter le monde, fût-il épique ou romanesque, que surgit la merveille, invitation à un autre système interprétatif, et à un autre système de valeurs, qu'elle qualifie en outre d'emblée de supérieurs.

Eléonore Andrieu Maître de Conférences en Langue et Littérature médiévales Université de Bordeaux/ EA TELEM elandrieu@wanadoo.fr

Maître de Conférences en Langue et littérature médiévales à l'Université de Bordeaux III. Domaines de recherche: historiographie médiévale au XII ${ }^{\mathrm{e}}$ siècle (Saint-Denis, domaine Plantagenêt) ; rapports entre les systèmes de pensée et pratiques d'écriture ecclésiastiques et les premiers textes littéraires en langue d'oïl du XII ${ }^{\mathrm{e}}$ siècle.

\section{Merveille and conflicts of values in the first texts in langue d'oil : some}

examples: We offer to examine the notions of merveilleux and merveille as devices conceived to confront different systems of value in the literary texts. Merveilleux and merveille may actually set up a polyphony because of the very essence of their "composante logico-sémantique", which, according to Jean-René Valette, points out "the discrepancy between a common experience or opinion, and the unexpected that actually occurs". Consequently, the three-fold ability of the character, accepting to wonder, perceiving the merveille, and not allowing his own system of interpretation to come to his aid, enables him to set out onto the Hero's path. However, during the period we are dealing with, the literary texts do not solely undertake to link the mirabilis and the ethical worth of the one who perceives, wonders and interprets : some contemporary Gregorian works use the mirabilis and the way it is perceived and interpreted, to dishonour the lay aristocracy and promote the clerici. Then, it might prove fruitful to confront the ecclesiastical propositions and the literary ones. From William of Orange to Calogrenant, the knightly characters facing the merveilleux would then embody some of the ecclesiastical views concerning the mirabilis; but also the capacity of the laity to reach a higher, and therefore spiritual, level of understanding. Perceiving the merveille would lead these characters not only to go into adventure, but also to switch from a discourse to another and from "mediocre" to superior ethics. More than merely disclosing "another world", merveilleux and merveille would set light upon rival discourses on the world and the conditions of a wonderful, yet possible encounter with the spiritual, of which the merveille would be the unmistakable evidence. 